\title{
Multicentre, prospective, open study to evaluate the safety and efficacy of hylan G-F 20 in knee osteoarthritis subjects presenting with pain following arthroscopic meniscectomy
}

\author{
J. P. Huskin · B. Vandekerckhove $\cdot$ P. Delincé $\cdot$ \\ R. Verdonk $\cdot$ J.-E. Dubuc $\cdot$ S. Willems $\cdot$ P. Hardy \\ F. J. Blanco $\cdot$ O. Charrois $\cdot$ F. Handelberg
}

Received: 15 January 2008/Accepted: 14 April 2008/Published online: 7 June 2008

(C) The Author(s) 2008

\begin{abstract}
The aim of the study was to evaluate the safety and efficacy of viscosupplementation with hylan G-F 20 in patients with mild to moderate osteoarthritis (OA) presenting with persistent knee pain 4-12 weeks after arthroscopic meniscectomy. A prospective, multi-centre, open study was carried out in patients with pain due to OA of the knee, not resolved by simple analgesics, 4-12 weeks after undergoing arthroscopic meniscectomy. To be eligible, patients had to score $\geq 50 \mathrm{~mm}$ and $\leq 90 \mathrm{~mm}$ on both walking pain and patient global assevssment visual analogue scales (VAS; $0-100 \mathrm{~mm}$ ) at baseline and be radiologically diagnosed pre-operatively with OA grade I or II on the Kellgren-Lawrence scale, with $<50 \%$ joint space narrowing. Patients received three intra-articular, $2 \mathrm{ml}$ injections of hylan G-F 20 in the target knee with an interval of 1 week between injections, and were followed for 52 weeks. The primary efficacy endpoint was the
\end{abstract}

J. P. Huskin

CHU Liège Sart-Tilman, Sce Orthopédie, B.35,

4000 Liege 1, Belgium

B. Vandekerckhove

A.Z. St-Jan, Orthopedie, Ruddershove 10,

8000 Brugge, Belgium

P. Delincé

CHU St-Pierre, 290 rue Haute, 1000 Brussels, Belgium

R. Verdonk

U.Z. Gent, 185 De Pintelaan, 9000 Ghent, Belgium

J.-E. Dubuc

Cliniques Universitaires Saint-Luc, Avenue Hippocrate 10,

1200 Brussels, Belgium

S. Willems

Clinique St Joseph Espérance, 75 Rue de Hesbaye,

4000 Liege, Belgium change from baseline in the walking pain VAS score at 26 weeks. Secondary outcome measures were the walking pain VAS scores at all other time points, the WOMAC Index at all time points, and patient and physician global assessment at all time points. The safety of the treatment was assessed using adverse event (AE) reports. A total of 62 patients (mean age 55.4 years, 52\% male) were enrolled. The mean walking pain VAS score decreased by $36.8 \mathrm{~mm}$ from baseline at 26 weeks $(P<0.0001)$, and also showed statistically significant decreases $(P<0.0001)$ at all other time points. The change in WOMAC total and subscale scores from baseline were statistically significant $(P<0.0001)$ at all time points, as were the decreases in the physician and patient global assessment VAS scores. There were 18 target knee AEs (mostly pain and/or swelling and/ or effusion) in 12 patients (19\%) considered to be at least possibly related to treatment. The majority of these (78\%)

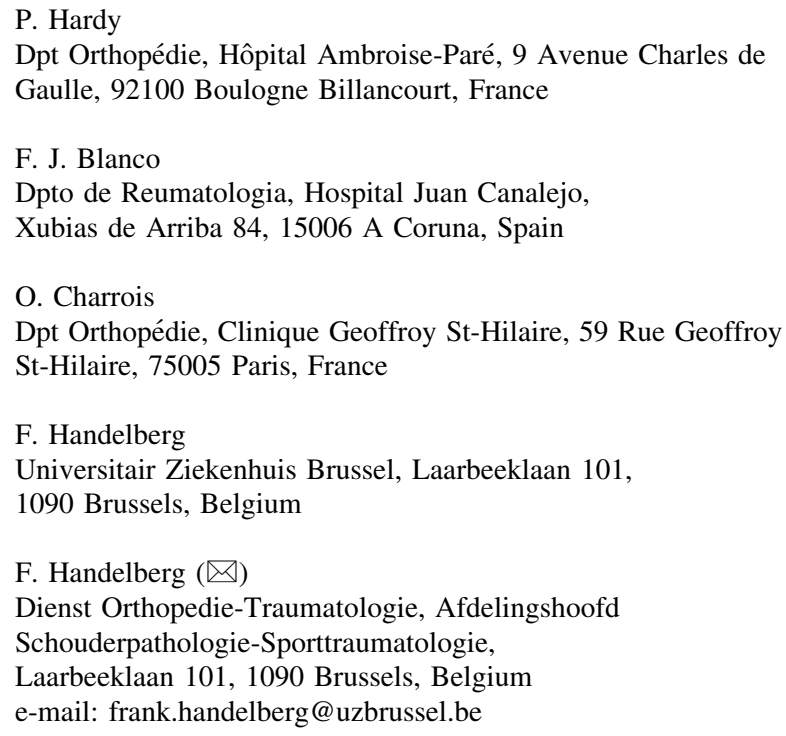


were mild or moderate in intensity. One patient (1.6\%) experienced a serious adverse event (synovitis) in the target knee that was considered possibly related to study treatment. Hylan G-F 20 provides effective pain relief and improves stiffness and physical function in patients with mild to moderate OA presenting with persistent osteoarthritic pain 4-12 weeks after arthroscopic meniscectomy. Symptomatic efficacy was maximised at 12 weeks and maintained at 26 and 52 weeks. The type (pain and/or swelling and/or effusion) and the intensity (mostly mild/ moderate) of AEs reported in this study are similar to those reported in other trials in different patient populations, but the incidence was higher (19\%). The risk/benefit of hylan G-F 20 in this particular population of patients is favourable.

Keywords Osteoarthritis - Arthroscopy · Hylan · Hyaluronan

\section{Introduction}

Osteoarthritis (OA) is a chronic, degenerative disorder associated with joint pain and loss of joint function. It is the most common disease to affect synovial joints and is one of the most frequently occurring chronic conditions.

The primary goal of treatment is the alleviation of pain, leading to an improvement in joint function and quality of life. Treatment options for knee OA include conservative therapies (education, weight loss, physical therapy, etc.), pharmacological therapies (simple analgesics, NSAIDs (including COX-2s)), intra-articular injections (corticosteroids, viscosupplements) and surgical intervention (e.g. arthroscopic debridement and lavage, meniscectomy, total knee replacement (TKR)).

More than $90 \%$ of patients with symptomatic OA of the knee have evidence of meniscal tears on magnetic resonance imaging [6], and arthroscopic meniscectomy can be used to treat patients who are unresponsive to more conservative therapies, but are not yet TKR candidates. However, the clinical benefit of arthroscopic debridement is unclear. In a double-blind study, Moseley et al. [11] randomised 180 patients who underwent arthroscopic debridement, arthroscopic lavage, or placebo surgery. At no point during the 24-month follow-up did either of the intervention groups report less pain or better function than the placebo group. In contrast, Matsusue [10] reported that $87 \%$ of patients with grade I or II OA achieved "excellent" symptomatic relief from meniscal tear debridement. Until such time as there is consensus on the clinical benefit of arthroscopic intervention for OA, it is likely that it will remain in the orthopaedic armamentarium. However, a sub-set of post-operative patients who have not gained adequate symptomatic relief will continue to present with persistent osteoarthritic pain several weeks or months after the procedure. The causative factors behind this persistent pain are unknown, but it is likely that the severity and extent of OA, the degree of debridement performed and the skill of the surgeon are major factors.

Viscosupplementation (intra-articular supplementation of hyaluronic acid) is a well-established treatment option in knee OA and is included in the professional guidelines for treatment of the disease in this joint $[2,12]$ Several metaanalyses have been conducted on the therapy class, which agree broadly that viscosupplementation is effective and safe $[3,5,16]$.

Hylan G-F 20 (Synvisc $^{\odot}$, Genzyme Corporation, MA, USA) is a high molecular weight, cross-linked derivative of hyaluronan. The safety and efficacy of viscosupplementation with hylan G-F 20 in OA of the knee is supported by prospective clinical trials $[1,13,14,17]$ and large retrospective studies $[8,15]$.

The use of viscosupplementation for the symptomatic relief of knee OA in patients after arthroscopic meniscectomy has not been widely studied. Marshall [9] reported on a retrospective series of 92 patients treated with hylan G-F 20 after failing conservative treatments and standardised arthroscopic debridement and injection of IA steroid. The majority of these patients $(77 \%)$ had "marked" or "severe" OA; $68 \%$ of patients did not go on to TKR during the 2.5 year follow-up and were considered as "salvaged".

This study aimed to assess the efficacy and safety of viscosupplementation with hylan G-F 20 in patients with mild or moderate knee OA who had failed to achieve adequate symptomatic relief from arthroscopic meniscectomy.

\section{Materials and methods}

\section{Patients}

Patients were enrolled at 10 centres across Europe: Belgium (7), France (2) and Spain. The study protocol, patient information and patient consent form were approved by the independent ethics committee of each investigating centre. Written, informed consent was obtained from each patient prior to enrolment in the study.

To be considered eligible for inclusion, patients had to present with osteoarthritic knee pain 4-12 weeks after arthroscopic meniscectomy (mean time from meniscectomy to the first injection of hylan G-F 20 was 53 days (range 27-107 days)) and must have had osteoarthritic cartilage lesion(s) observed during arthroscopy. The location and severity (Kellgren-Lawrence grade) of OA 


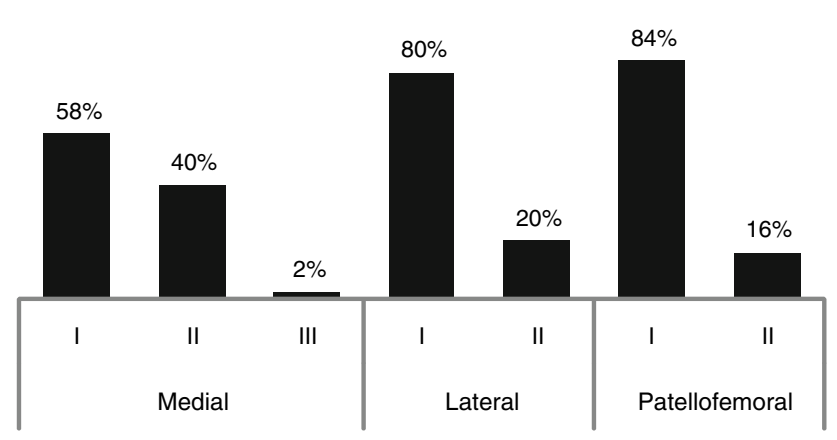

Fig. 1 OA location and radiological severity (Kellgren-Lawrence grade) at baseline (percentage of patients)

observed on baseline X-rays is shown in Fig. 1. Each arthroscopic surgery (Fig. 2) was performed by the authors, all skilled and experienced in this technique.

Patients were considered eligible for inclusion if they scored $\geq 50 \mathrm{~mm}$ and $\leq 90 \mathrm{~mm}$ on both the walking pain and patient global assessment visual analogue scales (VAS) $(0-100 \mathrm{~mm})$ at the screening visit. Patients were required to have X-ray evidence (pre-operative anteroposterior weight-bearing view (extension) + profile and patellofemoral at $30^{\circ}$ classical (taken within 3 months of the arthroscopic meniscectomy)) of osteoarthritis of the knee (Kellgren-Lawrence grade I or II, $<50 \%$ joint space narrowing).

The main exclusion criterion was any surgery on the cartilage during arthroscopic meniscectomy, except for non-therapeutic, superficial mechanical shaving of fibrillations and/or resection of unstable flaps in grade 2 and 3 lesions (grade 4 lesions were excluded), performed solely for the purpose of cleaning the area to enable accurate grading of the defect. Other exclusion criteria were: significant mechanical deficit (e.g. valgus/varus $>5 \%$ ); symptomatic contralateral knee OA; concomitant inflammatory joint disease (e.g. gout, rheumatoid arthritis); history of sepsis in any joint; prior viscosupplementation in the target knee; oral and/or parenteral glucocorticosteroids

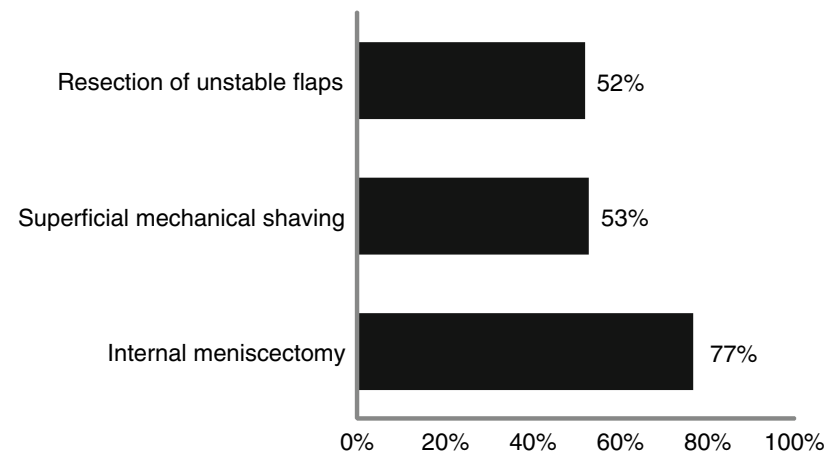

Fig. 2 Surgery performed at initial arthroscopy (percentage of patients) or intra-articular corticosteroids in any joint within 3 months prior to enrolment.

Trial design

This was a prospective, multi-centre, open study conducted between February 2002 and March 2005. A washout period of 1-3 weeks was applied (adapted to half-life), commencing at the screening visit, if patients had been taking prohibited analgesic medications. Once any required washout was completed and eligibility confirmed, patients presented for the baseline visit and received a single intraarticular $2 \mathrm{ml}$ injection of hylan G-F 20 using a strict aseptic administration technique. Prior to each injection, arthrocentesis was performed carefully, without injecting local anaesthetic into the joint space, to remove any effusion present. The suggested injection site was the lateral tibiofemoral joint space in the extended knee.

No standard rehabilitation protocol was imposed. Medication for pre-existing conditions was permitted. Also permitted were: paracetamol ( $\leq 4 \mathrm{~g} /$ day) except on the day before and on the day of the follow-up visits; analgesics and NSAIDs, other than COX-2 inhibitors, with a clearance time of $<8 \mathrm{~h}$ for pain other than OA in the study knee, or for post-injection pain management, but not on the day before or on the day of a study visit; aspirin $(\leq 325 \mathrm{mg}$ / day) for anti-thrombotic prophylaxis; topical corticosteroids for skin problems, except at the study knee; inhaled corticosteroids for asthma and allergic disease; non-pharmacological therapy (e.g. physical therapy) if begun before the study entry.

Prohibited medications were: analgesics and aspirin except as described above; COX-2 inhibitors; oral or parenteral corticosteroids; corticosteroid injections in any joint; topical analgesics at the study knee; therapeutic treatment with oral or parenteral anticoagulants. Chondroprotective agents were not prohibited, but were not used by any of the patients in the study. The use of all rescue and concomitant medications and therapies were recorded in the patient notes.

Patients returned to the investigational site at week 1 and week 2 for the remaining two injections of hylan G-F 20 , and for follow-up and safety evaluations at week 12, 26 and 52. At each visit, walking pain, physician global assessment and patient global assessment VAS were completed, the WOMAC $^{\mathrm{TM}}$ questionnaire was filled out by the patient, and concomitant therapy and adverse events were recorded.

Outcome measures

All efficacy analyses were performed on the intent-to-treat (ITT) population, which was defined as those patients who 
received at least one injection and for whom post-baseline efficacy data were present for at least the walking pain VAS. The primary efficacy outcome measure was the change from baseline in walking pain VAS at 26 weeks. Secondary efficacy outcome measures were: change from baseline in walking pain VAS at all other follow-up visits; change from baseline in WOMAC osteoarthritis index scores (pain (A), stiffness (B), physical functioning (C) and composite scores) at all time points; change from baseline in patient and physician global assessment VAS scores at all time points.

The safety assessment was based on reports of adverse events (AEs) collected between the signing of the informed consent form and the completion of the final follow-up visit for each patient.

\section{Results}

\section{Patients}

The ITT population consisted of 62 patients. During the study, seven patients were lost to follow-up, six withdrew due to target knee AEs, two became ineligible to continue, one withdrew consent and three patients discontinued from the study for other reasons. A total of 43 patients (69\%) completed the study. Baseline demographics for the ITT population are presented in Table 1 .

Primary efficacy endpoint

There was a statistically significant decrease in the mean score of the walking pain VAS from $65.0 \mathrm{~mm}$ at baseline to 28.0 at week $26(P<0.0001$; Fig. 3$)$.

Secondary efficacy endpoints

\section{Walking pain VAS}

The decreases from baseline in the walking pain VAS were statistically significant $(P<0.0001)$ at all time points (Fig. 3).

Table 1 Demographic data (ITT population)

\begin{tabular}{ll}
\hline Parameter & ITT population \\
\hline Number of patients & 62 \\
Gender: male/female, $n(\%)$ & $32 / 30(52 / 48)$ \\
Age (years): mean (SD) & $55.4(11.35)$ \\
Weight $(\mathrm{kg}):$ mean $(\mathrm{SD})$ & $81.6(14.97)$ \\
Height $(\mathrm{cm})$ : mean $(\mathrm{SD})$ & $168.8(9.64)$ \\
Body mass index $\left(\mathrm{kg} / \mathrm{m}^{2}\right):$ mean $(\mathrm{SD})$ & $28.9(6.26)$ \\
\hline
\end{tabular}

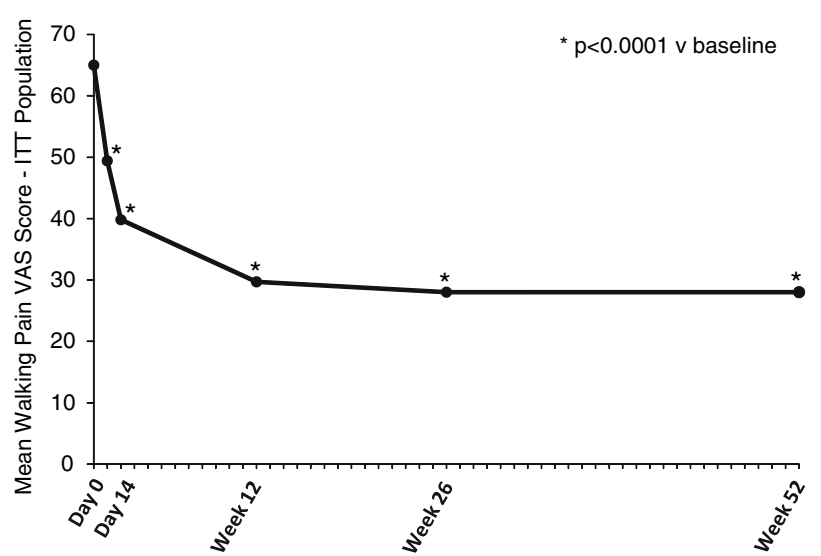

Fig. 3 Walking pain mean VAS score at each time point (ITT population)

\section{WOMAC Index}

Changes from baseline in the mean WOMAC scores (A, B, C and composite) were statistically significant $(P<0.0001)$ at all time points (Fig. 4).

\section{Patient and physician global assessment}

Changes from baseline in the mean patient and physician global assessment scores were statistically significant at all time points $(P<0.0001$; Fig. 5$)$.

\section{Safety}

Twelve patients (19\%) reported a total of 18 target knee AEs (arthralgia, joint swelling and joint effusion) that were considered possibly (10 AEs) or probably ( 8 AEs) related to the study treatment. These AEs were mostly considered to be mild (1 AE) or moderate (13 AEs) in severity. One serious target knee AE ("synovitis" i.e. pain and swelling,

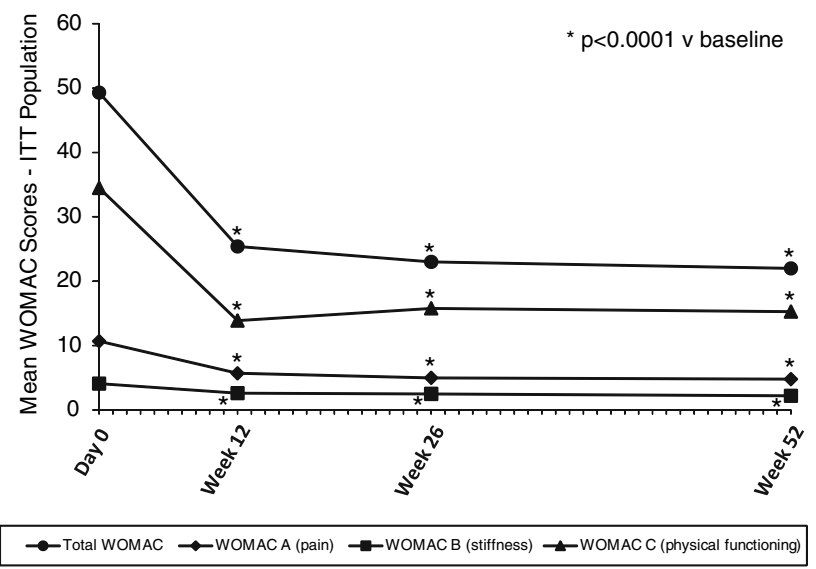

Fig. 4 Mean WOMAC Index scores at each time point (ITT population) 


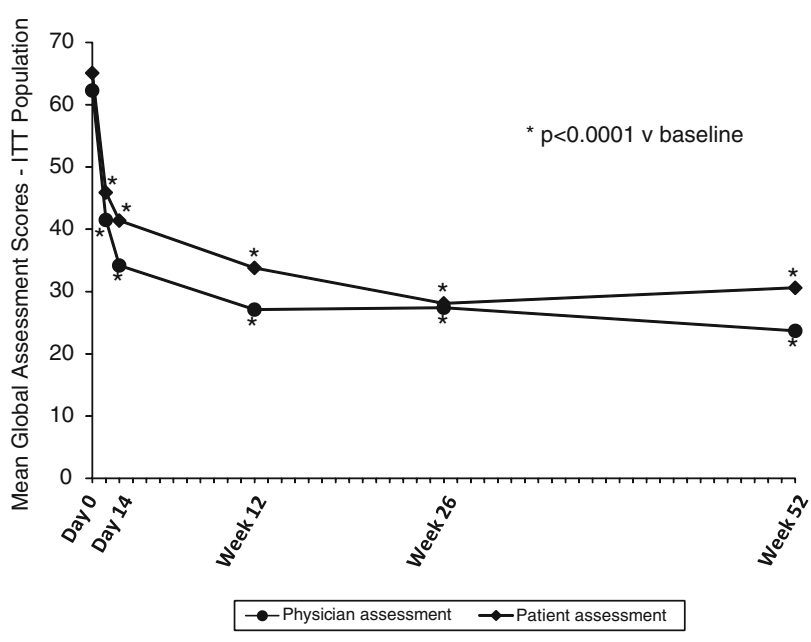

Fig. 5 Mean global assessment scores at each time point (ITT population)

which developed more than 2 months after the last injection of hylan G-F 20) was reported during the study and was considered possibly related to the study treatment.

Three patients discontinued the study due to the occurrence of target knee AEs considered possibly or probably study treatment related.

\section{Conclusions}

This is the first study to evaluate prospectively the efficacy and safety of intra-articular injections of hylan G-F 20 in patients with symptomatic knee OA unresponsive to simple analgesics 4-12 weeks after arthroscopic meniscectomy.

Although definitive conclusions about efficacy cannot be drawn from this study due to its open label design, hylan G-F 20 was effective at significantly reducing the pain associated with knee OA. Statistically significant changes from baseline were seen consistently in all primary and secondary efficacy outcome measures at all time points.

In the ITT population, reduction in pain was apparent from as soon as 7 days after injection, was maximised at 12 weeks and maintained at 26 and 52 weeks. These results reflect those reported in other studies in patients with knee OA, where the symptomatic relief obtained by patients outlasts substantially the product's residence time in the joint, thus supporting the hypothesis that viscosupplementation achieves its therapeutic effect by restoring the rheology of the joint temporarily [4].

The safety profile of hylan G-F 20 in knee OA is already supported by clinical literature. In the largest study conducted to date, Kemper et al. [7] assessed the safety of the treatment in 4,253 patients and reported AE rates of $4.2 \%$ of patients and $2.4 \%$ of injections. The types of AEs seen in our study were consistent with those seen in Kemper et al. and described in the product labelling (transient post-injection pain and/or swelling and/or effusion). The reason for the higher incidence of these AEs observed in this particular population of patients compared to previously published data is unknown. However, no new safety concerns were raised in this study and most AEs experienced were transient and of mild or moderate intensity.

We conclude that viscosupplementation with hylan G-F 20 has a favourable risk/benefit profile in patients with symptomatic knee OA presenting with persistent pain after arthroscopic meniscectomy.

Acknowledgment This study was sponsored by Genzyme Europe B.V.

Conflict of interest statement There are no conflicts of interest.

Open Access This article is distributed under the terms of the Creative Commons Attribution Noncommercial License which permits any noncommercial use, distribution, and reproduction in any medium, provided the original author(s) and source are credited.

\section{References}

1. Adams ME, Atkinson MH, Lussier AJ et al (1995) The role of viscosupplementation with hylan G-F 20 (Synvisc ${ }^{\circledR}$ ) in the treatment of osteoarthritis of the knee: a Canadian multicentre trial comparing hylan G-F 20 alone, hylan G-F 20 with nonsteroidal anti-inflammatory drugs (NSAIDs) and NSAIDs alone. Osteoarthr Cartil 3:213-225

2. American College of Rheumatology Subcommittee on Osteoarthritis Guidelines (2000) Recommendations for the medical management of osteoarthritis of the hip and the knee: 2000 update. Arthritis Rheum 43:1905-1915

3. Arrich J, Piribauer F, Mad P et al (2005) Intra-articular hyaluronic acid for the treatment of osteoarthritis of the knee: systematic review and meta-analysis. CMAJ 172:1039-1043

4. Bagga H, Burkhardt D, Sambrook P et al (2006) Long- term effects of intraarticular hyaluronan on synovial fluid in osteoarthritis of the knee. J Rheumatol 33:946-950

5. Bellamy N, Campbell J, Robinson V et al (2006) Viscosupplementation for the treatment of osteoarthritis of the knee. Cochrane Database of Systematic Reviews Issue 2. Art. No.: CD005321

6. Bhattacharyya T, Gale D, Dewire P et al (2003) The clinical importance of meniscal tears demonstrated by magnetic resonance imaging in osteoarthritis of the knee. J Bone Joint Surg Am 85-A(1):4-9

7. Kemper F, Gebhardt U, Meng T et al (2005) Tolerability and short-term effectiveness of hylan G-F 20 in 4253 patients with osteoarthritis of the knee in clinical practice. Curr Med Res Opin 21:1261-1269

8. Lussier A, Cividino AA, McFarlane CA et al (1996) Viscosupplementation with hylan for the treatment of osteoarthritis: findings from clinical practice in Canada. J Rheumatol 23:15791585

9. Marshall KW (1999) Hylan G-F 20 (Synvisc $^{\circledR}$ ) salvage of patients failing a standardised arthroscopic debridement treatment 
algorithm. Poster presented at the 9th conference of the Orthopaedic Research Society International, 3-4 June 1999, Brussels, Belgium

10. Matsusue Y, Thomson NL (1996) Arthroscopic partial medial meniscectomy in patients over 40 years old: a 5- to 11-year follow-up study. Arthroscopy 12(1):39-44

11. Moseley JB, O'Malley K, Petersen NJ et al (2002) A controlled trial of arthroscopic surgery for osteoarthritis of the knee. N Engl J Med 347:81-88

12. Pendleton A, Arden N, Dougados M et al (2000) EULAR recommendations for the management of knee osteoarthritis: report of a task force of the Standing Committee for International Clinical Studies Including Therapeutic Trials (ESCISIT). Ann Rheum Dis 59:936-944

13. Raynauld JP, Torrance GW, Band PA et al (2002) A prospective, randomised, pragmatic, health outcomes trial evaluating the incorporation of hylan G-F 20 into the treatment paradigm for patients with knee osteoarthritis (Part 1 of 2): clinical results. Osteoarthr Cartil 10:506-517

14. Scale D, Wobig M, Wolpert W (1994) Viscosupplementation of osteoarthritic knees with hylan: a treatment schedule study. Curr Ther Res 55:220-232

15. Waddell DD, Bricker DC (2006) Clinical experience with the effectiveness and tolerability of hylan G-F 20 in 1047 patients with osteoarthritis of the knee. J Knee Surg 19:19-27

16. Wang CT, Lin J, Chang CJ et al (2004) Therapeutic effects of hyaluronic acid on osteoarthritis of the knee. A meta-analysis of randomized controlled trials. J Bone Joint Surg Am 86-A:538545

17. Wobig M, Dickhut A, Maier R et al (1998) Viscosupplementation with hylan G-F 20: a 26-week controlled trial of efficacy and safety in the osteoarthritic knee. Clin Ther 20:410-423 